\title{
A Study on-effect of Hematoma and Perihematomal Edema Volume on GCS at the time of admission in Patients with Spontaneous Lobar and Basal Ganglia Hemorrhage
}

Joarder $\mathrm{MA}^{1}$, Karim $\mathrm{AKMB}^{2}$, Barua $\mathrm{KK}^{3}$, Hossain $\mathrm{MA}^{4}$

\begin{abstract}
Objective: To know effect of hematoma and perihematomal edema volume on Glasgow Coma Scale (GCS) at the time of admission in patients with spontaneous lobar and basal ganglia hemorrhage. Methods: this cross sectional study was conducted in Neurosurgery department, BSMMU to observe effect of hematoma and perihematomal edema volume on GCS at the time of admission in patients with spontaneous lobar and basal ganglia hemorrhage. The duration of study period was from November 2005 to April 2007. Results: a total 48 patients were included in the study and the variables that were analyzed included site of hematoma, volume of hematoma, perihematomal edema and the initial GCS score. Associations between the hematoma and perihematomal edema, site of hemorrhage and perihematomal edema, hematoma volume and GCS at the time of admission, perihematomal edema volume and GCS at the time of admission were evaluated. Level of consciousness (GCS) at the time of admission is the key factor in predicting outcome and neurological deterioration. In our study we found significant association between- hematoma and perihematomal edema volume, hematoma volume and GCS at the time of admission, perihematomal edema volume and GCS at the time of admission. But there was no significant association found between hemorrhage site and perihematomal edema.Conclusion: more the volume of hematoma more was the volume of perihematomal edema. More the volume of hematoma less was the GCS. More the volume of perihematomal edema less was the GCS. But we found no significant association between site of hemorrhage and perihematomal edema volume. GCS is an established predictor of outcome in patients with SICH (spontaneous intracerebral hemorrhage). Accurate prediction of the outcome in ICH patients is important for several reasons: a reliable prognosis must be given to patient and relatives as soon as possible, realistic rehabilitation goals should be set and resources should be allocated in the most efficient way.
\end{abstract}

\section{Introduction}

Spontaneous intracerebral hemorrhage is defined as bleeding into brain parenchyma without accompanying trauma. Spontaneous ICH results from an intracerebral arterial or, less frequently, venous rupture, which leads to the formation of an intraparenchymal hematoma. The hematoma expands following the path of least resistance, usually along white matter tracts, and occasionally dissects its way into the ventricular system. In time the bleeding slows and eventually stops as increasing tissue

1. Specialist, Department of Neurosurgery, Apollo Hospitals Dhaka. 2. Registrar, Department of Neurosurgery, Apollo Hospitals Dhaka. 3. Professor, Department of Neurosurgery, BSMMU, Dhaka. 4. Professor and Chairman, Department of Neurosurgery, BSMMU, Dhaka. 
A Study on-effect of Hematoma and Perihematomal Edema Volume on GCS at the time of admission in Patients with Spontaneous Lobar and Basal Ganglia Hemorrhage

pressure leads to tamponade of the rupture site. Neurologic deficit results from both direct tissue destruction and indirect compression of neural structures, usually in proportion to both the volume of the hematoma and its rate of expansion. ICH can occur at any site within the brain, though some areas are more susceptible than others. Eighty percent occur within the cerebral hemispheres while the remaining $20 \%$ are infratentorial. Of note, hypertensive bleeds occur in the deep gray matter $(65 \%)$, pons $(11 \%)$, and cerebellum (8\%), whereas bleeds associated with other disorders are likely to be located in the sub cortical white matter (45\%), deep gray matter $(36 \%)$, pons $(10 \%)$, and cerebellum (3\%). Similarly, bleeds at any one particular location tend to be associated with certain conditions more than others (Martin and Holland 2005).

For example, lobar bleeds - found in the subcortical white matter - are often associated with tumors, vascular malformations, and cerebral amyloid angiopathy; basal ganglia bleeds, which may extend to include the internal capsule and thalamus, are often associated with hypertension as are brain stem bleeds, though these are usually much more serious. Finally, cerebellar bleeds tend to accompany tumors, vascular malformations, blood dyscrasias, and hypertension (Martin and Holland 2005).

An SICH can be classified as either primary or secondary depending on the underlying cause of the hemorrhage. Primary ICH accounts for approximately 70 to $80 \%$ of cases and is due to spontaneous rupture of small vessels damaged by hypertension or amyloid angiopathy. Secondary ICH is associated with a number of congenital and acquired conditions such as vascular anomalies, coagulopathies, tumours, and various drug therapies. Cerebrovascular damage to small arteries and arterioles due to chronic hypertension is recognized as the most significant cause of primary SICH. Cerebral amyloid angiopathy is the other major cause of primary SICH and an important cause of lobar $\mathrm{SICH}$ in elderly populations. Vascular anomalies are the second most common cause of SICH overall. Aneurysms, AVMs, cavernomas, duralarteriovenous fistulas, and venous malformations all can result in secondary SICH. The hemorrhage due to a ruptured aneurysm almost always has a subarachnoid component and often extends into the ventricles. There should be a high index of suspicion in young patients with frontal or temporal lobe clots. Arteriovenous malformations are associated with an estimated mean annual hemorrhage risk of $4 \%$ (Fewel et al. 2003). The worldwide incidence of intracerebral hemorrhage ranges from 10 to 20 cases per 100,000 population and increases with age. Intracerebral hemorrhage is more common in men than women, particularly those older than 55 years of age (Qureshi et al. 2001).

Spontaneous intracerebral haemorrhage accounts for approximately $4-14 \%$ of all strokes and is associated with high mortality and morbidity. Between $32 \%$ and $50 \%$ of patients die within the first month, and only $20 \%$ are independent six months after intracerebral bleeding. Several clinical and 


\section{ORIGINAL ARTICLE}

radiological factors such as age, level of consciousness, hypertension, volume of the hematoma, volume of peripheral edema, midline shift displacement on initial computed tomography (CT), and intraventricular spread of the bleeding appear to be markers of poor prognosis after spontaneous ICH (Castellanos et al. 2005). Accurate prediction of the outcome in ICH patients is important for several reasons: a reliable prognosis must be given to patient and relatives as soon as possible, realistic rehabilitation goals should be set and resources should be allocated in the most efficient way. Several studies have identified individual clinical and radiological variables that are correlated with mortality in ICH patients. These include hematoma size or volume, presence of hydrocephalus, intraventricular extension, midline shift, level of consciousness, age, fever, hyperglycemia and pulse pressure (Hallevy et al. 2002).

\section{Materials and Methods}

This cross sectional study was conducted in Neurosurgery department, BSMMU to observe effect of hematoma and perihematomal edema volume on GCS at the time of admission in patients with spontaneous lobar and basal ganglia hemorrhage. The duration of study period was from November 2005 to April 2007. The study was done on admitted patients with a CT scan confirmed spontaneous intracerebral hemorrhage fulfilling selection criteria. All these patients were evaluated on the basis of history, clinical examination and CT scan findings.
Inclusion criteria are-1. Compatible history. 2 . Patients admitted with CT scan evidence of basal ganglia and lobar SICH. 3. Patients who agreed to be included in this study. Exclusion criteria are-1. ICH with thalamic hemorrhage or intraventricular extension. 2. Infratentorial ICH. 3. Spontaneous ICH with subarachnoid hemorrhage. 4. Those patients who were on anticoagulant therapy. 5. History of trauma. The sample size $(\mathrm{N})$ was 48 . The volume of the hematoma and edema was measured by modified ellipsoid formula simply by \pm 6 ABC which practically amounts to $1 / 2 \mathrm{ABC}$ (Kothari et al. 1996). Perihematomal edema volume (rim of hypodensity) was calculated by subtracting the hematoma volume from the combined hematoma and perihematomal edema volume (McCarron et al.). CT scan was examined and hematoma and perihematomal edema volume were recorded.

\section{Results}

During this period, 48 patients were included in the study. Their mean age was 58 years (range $35-80$ years), 33 (69\%) patients were male and $15 \quad(31 \%) . \quad 25 \quad(52 \%)$ patients were hypertensive, 5 (10\%) patients were diabetic, 8 (17\%) patients were having both DM and HTN and $10(21 \%)$ patients were non DM \& non HTN. The ICH was lobar in 17 (35\%) patients, basal ganglia in $25(52 \%)$ and both in $6(13 \%)$. Mean volume of hematoma was $41 \mathrm{ml}$ (range14-90ml). Mean volume of perihematomal edema was $15 \mathrm{ml}$ (range 3-31ml). Mean GCS was 8 (range 3-15). 
A Study on-effect of Hematoma and Perihematomal Edema Volume on GCS at the time of admission in Patients with Spontaneous Lobar and Basal Ganglia Hemorrhage

Table I: Association between hematoma volume and perihematomal edema volume.

\begin{tabular}{|l|c|c|c|c|}
\hline \multicolumn{2}{|c|}{} & \multicolumn{2}{c|}{ Edema volume range } & Total \\
\hline \multicolumn{2}{|l|}{} & $\leq 15$ & $>15$ & \\
\hline \multirow{2}{*}{ Hematoma volume range } & $\leq 40$ & 26 & 5 & 31 \\
\cline { 2 - 5 } & $>40$ & 1 & 16 & 17 \\
\hline \multicolumn{2}{|l|}{ Total } & 27 & 21 & 48 \\
\hline
\end{tabular}

Pearson Chi-Square $(\mathrm{x} 2)$ test value $27.13 \mathrm{p}$ value $=<.001$ ( $\mathrm{p}$ value $<.05$ was considered significant)

Table II: Association between hemorrhage site and perihematomal edema volume.

\begin{tabular}{|c|c|c|c|c|}
\hline \multicolumn{2}{|c|}{} & \multicolumn{2}{c|}{ Edema volume range } & Total \\
\hline \multicolumn{2}{|c|}{ Hemorrhage site } & $<15$ & $>15$ & \\
\hline & Lobar & 8 & 9 & 17 \\
\hline & Basal ganglia and both & 19 & 12 & 31 \\
\hline \multicolumn{2}{|c|}{ Total } & 27 & 21 & 48 \\
\hline
\end{tabular}

Pearson Chi-Square $( \pm 2)$ test value 0.904 . $p$ value $=>0.1$ ( $p$ value $<0.05$ was considered significant)

Table III: Association between hematoma volume and GCS at the time of admission.

\begin{tabular}{|l|l|c|c|c|}
\hline \multicolumn{2}{|c|}{} & \multicolumn{2}{|c|}{ Hematoma volume range } & Total \\
\cline { 3 - 5 } \multicolumn{2}{|c|}{} & $\leq 40$ & $>40$ & \\
\hline \multirow{2}{*}{ GCS Range } & $\leq 8$ & 11 & 15 & 26 \\
\cline { 2 - 5 } & $>8$ & 20 & 2 & 22 \\
\hline Total & 31 & 17 & 48 \\
\hline
\end{tabular}

Pearson Chi-Square $( \pm 2)$ test value 12.30. $p$ value $=<0.001$ ( $p$ value $<0.05$ was considered significant).

Table IV: Association between perihematomal edema volume and GCS at the time of admission

\begin{tabular}{|l|c|c|c|c|}
\hline \multicolumn{2}{|c|}{} & \multicolumn{2}{|c|}{ Edema volume range } & \multirow{2}{*}{ Total } \\
\cline { 3 - 4 } \multicolumn{2}{|c|}{} & $\leq 15$ & $>15$ & \\
\hline \multirow{2}{*}{ GCS Range } & $\leq 8$ & 9 & 17 & 26 \\
\cline { 2 - 4 } & $>8$ & 18 & 4 & 22 \\
\hline Total & 27 & 21 & 48 \\
\hline
\end{tabular}

Pearson Chi-Square $(\chi 2)$ test value 10.79. $p$ value $=0.001$ ( $p$ value $<0.05$ was considered significant). 


\section{ORIGINAL ARTICLE}

\section{Discussion}

In our series the mean age (58.50 years) was nearer to the mean age of the study of Zazulia et al 1999 (61.50 years) but much lower than that of the study of Bilbao et al 2005 (69 years) in Spain. The age group with highest frequency was also lower level in our series than that of the series of Bilbao et al. These observations reflect the higher life expectancy in western people.

The male to female ratio was higher in our study (2.19:1) than other (Bilbao et al. 1.83:1, Ahmed et al. 1.38:1) probably due to the fact that the male bed number of department of Neurosurgery, BSMMU, is 3 times greater than that of the female and males are more privileged in our society.
In our series $52 \%$ of the patients were hypertensive which were almost similar with the study of Zazulia et al 1999, they found $54 \%$ of patients with hypertension. But our findings did not correlate with the findings of Hallevy et al (2002) as $72.8 \%$ of their patients were hypertensive.

Diabetes mellitus was found in $10 \%$ of patient in our series which was comparable with the findings of Juvela et al (1995) as they found $8.97 \%$ of their cases had DM. But our findings greatly differ from the findings of Hallevy et al (2002) as they found 23.9\% of the patients had DM. Our findings were also nearer the findings of Arbix et al (2000).They found $15.3 \%$ of the patient with DM.

\begin{tabular}{|c|c|c|c|}
\hline Studies & Yen et al 2005 & $\begin{array}{c}\text { Razzaq and Hussain } \\
1999\end{array}$ & Present study \\
\hline $5-8$ & $32.05 \%$ & $34.24 \%$ & $37.5 \%$ \\
\hline $9-12$ & $21.02 \%$ & $45.2 \%$ & $39.6 \%$ \\
\hline$>13$ & $46.92 \%$ & $16.44 \%$ & $6.3 \%$ \\
\hline
\end{tabular}

In our series we found GCS range 3-15, our findings were compared with the findings of other studies demonstrated in tabulated form below:

In our series we found most of the hemorrhage site are in the basal ganglia $(52 \%)$ region as most of the patients were hypertensive, $36 \%$ hemorrhage were in the lobar region and $12 \%$ hemorrhage were occupying both in the lobar and basal ganglia region. Our study were nearer to the study of Zazulia et al 1999 (lobar 25\%, basal ganglia 51.3\%) but far different from study done by McCarron et al 2005. They found $43.2 \%$ were in the basal ganglia and $56.8 \%$ were in the lobar region.

In this study range of the hematoma volume were 6 to $90 \mathrm{ml}$. Mean hematoma volume was $41 \mathrm{ml}$. Maximum hematoma volume (65\%) were below $40 \mathrm{ml}$ and $35 \%$ were found above $40 \mathrm{ml}$.

In our series we found mean perihematomal edema volume were $15 \mathrm{ml}$. Range of 


\begin{tabular}{|c|c|}
\hline Carhupoma et al 2003 & Present study \\
\hline $\mathrm{p}=0.001$ & $\mathrm{p}=<0.001$ \\
\hline
\end{tabular}

perihematomal edema volume were 3-31 ml. Maximum edema volume (56\%) were below 15 $\mathrm{ml}$ and $44 \%$ were above $15 \mathrm{ml}$.
In our study there was direct relationship between hematoma volume and perihematomal edema volume. Comparison with other study

\begin{tabular}{|c|c|}
\hline McCarron et al. (2005) & Present study \\
\hline $\mathrm{p}=0.71$ & $\mathrm{p}=>0.1$ \\
\hline
\end{tabular}

given below:

In our study association between the hematoma and perihematomal edema volume was found significant $(\mathrm{p}=<0.001)$ in $95 \%$ confidence limit. Our finding was similar to the finding of Carhupoma et al $2003(\mathrm{p}=0.001)$. More the volume of hematoma more will be the volume of perihematomal edema.

Comparison of findings of association between the hemorrhage site and perihematomal edema volume presented in tabulated form below:

Our observation was similar to the observation of McCarron et al. 2005. The association between hemorrhage site and perihematomal edema volume was not found significant $(\mathrm{p}=$ $>0.1$ ). So there was no relationship between hemorrhage site and edema volume.

It was observed that the volume of hematoma is a very important factor which affects the GCS in patients with SICH at the time of admission. In 2001 Qureshi et al. found that patients with a large hematoma usually have a decreased level of consciousness (GCS) at presentation as a result of increased intracranial pressure and the direct compression or distortion of the thalamic and brain-stem reticular activating system. Our observation was found similar to the Qureshi et al. 2001. The volume of hematoma has been found to correlate with an individual's level of consciousness (GCS). In our study there was an inverse relationship between hematoma volume and GCS at the time of admission. We found significant association $(\mathrm{p}=<-0.001)$ between the volume of hematoma and GCS. More the volume of hematoma less was the GCS.

Though very few study has been done on the effect of perihematomal edema volume in patients with SICH. Zazulia et al 1999 found that perihematomal edema volume affects the GCS by producing mass effect. In this study we found negative correlation between perihematomal edema volume and GCS. There was significant association $(p=0.001)$ in $95 \%$ confidence limit between perihematomal edema volume and GCS.

\section{Summary}

Level of consciousness (GCS) at the time of admission is the key factor in predicting outcome and neurological deterioration. There are few established factor which acts as a 


\section{ORIGINAL ARTICLE}

predictor of outcome in spontaneous intracerebral hemorrhage by affecting the GCS of patients at the time of admission. In our study we found significant association between-

- Hematoma and perihematomal edema volume $(\mathrm{p}=<0.001)$.

- Hematoma volume and GCS at the time of admission $(\mathrm{p}=<0.001)$

- Perihematomal edema volume and GCS at the time of admission $(\mathrm{p}=0.001)$.

But there was no significant association found between hemorrhage site and perihematomal edema $(\mathrm{p}=>0.1)$.

\section{Conclusion}

GCS is an established predictor of outcome in patients with SICH. Accurate prediction of the outcome in $\mathrm{ICH}$ patients is important for several reasons: a reliable prognosis must be given to patient and relatives as soon as possible, realistic rehabilitation goals should be set and resources should be allocated in the most efficient way.

\section{References}

1. Arbix A, Massons J, Garcia-Eroles L, Oileeres M, Trga C. Diabetes is an independent riskfactor for in-hospital mortality from acute spontaneous intracerebral hemorrhage. Diabetes care. 2000. vol 23 p. 1527-32.

2. Bilbao G, Garibi J, Pomposo I, Pijoan JI, Carrasco A et al. A prospective study of a series of 356 patients with supratentorial spontaneous intracerebral hematomas treated in a neurosurgical department. ActaNeurochir (Wein). 2005. vol 147 p. 823-29.
3. Carhupoma JR, Hanley D F, Banrjee M, Beauchamp NJ. Brain edema after human cerebral hemorrhage: A Magnetic resonance imaging volumetric analysis. Journal of neurosurgical anesthesiology. 2003;15(3): p. 691-95.

4. Castellanos M, Leira R, Tejada J, Gil-Peralta A, Da vlos A, Castillo J. Predictors of good outcome in medium to large spontaneous supratentorial intracerebral haemorrhages. J Neurol Neurosurgery Psychiatry. 2005;76:691-95.

5. Fewel ME, ThompsonBG, Hoff JT. Spontaneous intracerebral hemorrhage: a review. Neurosurg Focus. 2003;15(4): Article 1

6. Hallevy C, Ifergane G, Kordysh E, Herishanu Y. Spontaneous supratentorial intracerebral hemorrhage: criteria for short-term functional outcome prediction. $\mathrm{J}$ Neurol. 2002;249:170470-19.

7. Juvela S, Hillbom M, Palomaki H. Risk factors for spontaneous intracerebral hemorrhage. Stroke. 1995;26: p. $1558-64$.

8. Kothari RU, Brott T, Broderick JP, Barsan WG, Sauerbeck LR, Zuccarello M. et al. The ABCs of measuring intracerebral hemorrhage volumes. Stroke.1996;27:1304-05

9. Martin NA, Holland MC. Rengachary SS, Ellenbogen RG, editors. Spontaneous intracerebral hemorrhage. Principle of neurosurgery 2nd ed. Elsevier Mosby; 2005. p. 261-69.

10. McCarron MO, Hoffmann KL, DeLong DM. Intracerebral hemorrhage outcome: apolipoprotein $\mathrm{E}$ genotype, hematoma, and edema volumes.

Neurology.1999;53:2176-9.

11. McCarron MO, McCarron P, Alberts MJ. Location characteristics of early peri hematomal edema. J of Neurology Neurosurgery Psychiatry. 2006;77:378-80. 12. Qureshi AI, Tuhrim S, Broderick JP, Batjer HH, Hondo H, Hanley DF. Spontaneous intracerebral hemorrhage. N Engl J Med. 2001;344(19):1450-60. 13. Razzaq AA, Hussain R. Determinants of 30-day mortality of spontaneous intracerebral hemorrhage in Pakistan. Surg Neurol. 1998;50: p. 336-43.

14. Zazulia AR, Diringer MN, Derdeyn CP, Powers WJ. Progression of mass effect after intracerebral hemorrhage. Stroke. 1999;30:1167-73. 